Journal of Magnetism and Magnetic Materials 9 (1978) 83-85

๑ North-Holland Publishing Company

\title{
TWO MAGNON RESONANT RAMAN SCATTERING IN TRANSITION METAL OXIDES
}

\author{
R. MERLIN, T.P. MARTIN, A. POLIAN *, M. CARDONA, \\ Max-Planck-Institut für Festkörperforschung, 7000 Stuttgart 80, Fed. Rep. Germany
}

\section{B. ANDLAUER,} Institut für Angewandte Festkörperphysik der Fraunhofer Gesellschaft, 7800 Freiburg, Fed. Rep. Germany

and

D. TANNHAUSER, Technion-Israel Institute of Technology, Department of Physics, Iaifa, Israel

Received 23 March 1978

Resonant Raman scattering by two magnons is reported for the ${ }^{6} \mathrm{~A}_{1} \rightarrow{ }^{4} \mathrm{~T}_{2}$ absorption band of $\alpha-\mathrm{Fe}_{2} \mathrm{O}_{3}$ but is absent for the corresponding band of $\mathrm{FeBO}_{3}$. This is related to the absence of inversion symmetry in the former and its presence in the latter compound. The absorption edge of $\mathrm{NiO}$ at $\approx 3.6 \mathrm{eV}$ shows strong resonance for phonons but not for magnon processes. From this fact it is conjectured that the edge is due to $\mathrm{O}(2 \mathrm{p}) \rightarrow \mathrm{Ni}(3 \mathrm{~d})$ transitions.

In this letter we present results on the resonant scattering of light by two magnons in the antiferromagnetic transition metal compounds $\alpha \cdot \mathrm{Fe}_{2} \mathrm{O}_{3}, \mathrm{FeBO}_{3}$ and $\mathrm{NiO}$.

The optical properties of $\mathrm{FeBO}_{3}$ have been well studied [1]. There is a broad absorption band centered at $2.05 \mathrm{eV}$ due to transitions from the ${ }^{6} \mathrm{~A}_{1 \mathrm{~g}}\left({ }^{6} \mathrm{~S}\right)$ to the ${ }^{4} \mathrm{~T}_{2 \mathrm{~g}}\left({ }^{4} \mathrm{G}\right)$ manifolds [1]. These transitions occur at $1.98 \mathrm{eV}$ in $\alpha-\mathrm{Fe}_{2} \mathrm{O}_{3}$ [2]. The two-magnon spectra of both compounds are also well known $[3,4]$. We have measured the intensity of the two-magnon scattering as a function of laser frequency. The results for $\alpha-\mathrm{Fe}_{2}-\mathrm{O}_{3}$ are shown in fig. 1. The corresponding results for $\mathrm{FeBO}_{3}$ are not shown because the two-magnon Raman scattering cross section is nearly a constant in the same optical region.

\footnotetext{
* Alexander von Humboldt fellow, present address: Laboratoire de Physique des Solides, Université Pierre et Maric Curie, 75230 Paris-Cedex 05, France.
}

The cross section of the two-magnon line can be obtained following Loudon's derivation [5]. The corresponding scattering tensor has the form

$$
\begin{aligned}
& R_{k l}=\sum_{\substack{\mu \nu \\
\eta \rightarrow 0^{+}}}\left\langle\phi_{\mathrm{i}} \uparrow \phi_{j} \downarrow\left|e r_{k}\right| \phi_{i} \uparrow \phi_{\nu} \downarrow\right\rangle \\
& \quad \times\left\langle\phi_{i} \uparrow \phi_{\nu} \downarrow\left|\frac{e^{2}}{r}\right| \phi_{j} \uparrow \phi_{\mu} \downarrow X \phi_{j} \uparrow \phi_{\mu} \downarrow l e r_{l} \mid \phi_{j} \uparrow \phi_{i} \downarrow\right\rangle \\
& \quad /\left(E_{\nu}-\omega-i \eta\right)\left(E_{\mu}+\Omega-\omega-i \eta\right),
\end{aligned}
$$

where $\phi_{i}$ and $\phi_{j}$ are the ground state orbitals of ions $i$ and $j, \phi_{\nu}$ and $\phi_{\mu}$ are the corresponding excited state orbitals with energies $E_{\nu}$ and $E_{\mu}$. The laser energy is denoted by $\omega$, the two-magnon energy by $\Omega, e r_{k, l}$ are the components of the electric dipole operator. If one confines the summation to nearly degenerate intermediate states $\left(E_{\nu} \simeq E_{\mu}\right)$ and, moreover, if the exchange matrix element is assumed isotropic and independent of the intermediate state, it is possible to transform 


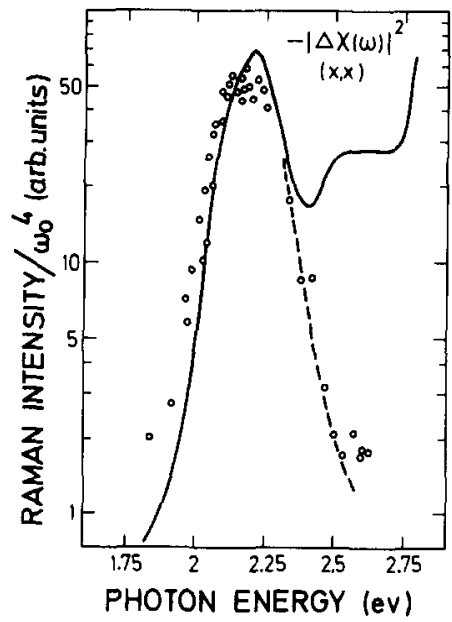

Fig. 1. Dependence of the integrated intensity of the twomagnon Raman scattering in $\alpha-\mathrm{Fe}_{2} \mathrm{O}_{3}$ on the laser frequency at $T=300 \mathrm{~K}$ for $\mathrm{z}(\mathrm{xx}) \overline{\mathrm{z}}$ configuration. The solid line represents $|[x(\omega)-\chi(\omega-\Omega)] / \Omega|^{2}$ where $x$ is the electric susceptibility.

eq. (1) into an expression containing only the dielectric susceptibility. Some algebraic manipulations then yield [6]:

$R_{k l} \approx \omega^{2} \frac{\chi_{k l}(\omega)-\chi_{k l}(\omega-\Omega)}{\Omega}$,

where $\chi$ is the electric susceptibility. The scattering cross section $\sigma$ is proportional to the square of the tensor $R_{k l}$ contracted with the polarization vectors of the incident and scattered fields.

Eq. (2) was evaluated and the resulting cross section is compared with the experimental one in fig. 1 . The experimental points fit very well the first peak in the calculated spectrum at $2.2 \mathrm{eV}$. The dipole transitions above $2.7 \mathrm{eV}$ contribute very little to the resonant Raman scattering.

The ${ }^{6} \mathrm{~S} \rightarrow{ }^{4} \mathrm{G}$ transitions are spin and parity forbidden. The spin-orbit coupling removes, in principle, the spin restriction. Moreover, the $\mathrm{Fe}^{3+}$ site in $\alpha-\mathrm{Fe}_{2} \mathrm{O}_{3}$ lacks inversion symmetry [7]. This means that the parity selection rule is lifted (possibly through admixture of the $3 \mathrm{~d}$ states of $\mathrm{Fe}$ with $2 \mathrm{p}$ states of oxygen). This is not the case in $\mathrm{FeBO}_{3}$ where the $\mathrm{Fe}^{3+}$ environment has inversion symmetry [1]. The ${ }^{6} \mathrm{~A}_{1} \rightarrow{ }^{4} \mathrm{~T}_{2}$ transition is then electric dipole allowed in $\alpha-\mathrm{Fe}_{2} \mathrm{O}_{3}$ but not in $\mathrm{FeBO}_{3}$. This explains why one does observe a resonance in the two-magnon cross section in one case but not in the other. Correspondingly, the absorp- tion coefficient of $\alpha-\mathrm{Fe}_{2} \mathrm{O}_{3}$ is about one order of magnitude larger than that of $\alpha-\mathrm{Fe}_{2} \mathrm{O}_{3}$ in the region of the ${ }^{6} \mathrm{~S} \rightarrow{ }^{4} \mathrm{G}$ transitions $[1,8]$. It is doubtful, however, whether spin-orbit coupling and the breakdown of inversion symmetry alone can account for the strength of the observed absorption in $\alpha-\mathrm{Fe}_{2} \mathrm{O}_{3}\left(\approx 10^{3} \mathrm{~cm}^{-1}\right)$ $[8,9]$.

Generally, one would expect to observe a resonance in the exchange induced scattering by magnons only for an electric dipole allowed transition whose initial state is that of a magnetic ion. Consequently, we believe that the higher energy transitions in fig. 1 involve the $2 p$ states of oxygen as initial states. Such transitions will be dipole allowed but will not make a strong contribution to the resonant magnon scattering.

We have also measured the dependence on exciting energy of the two-magnon Raman scattering cross section in $\mathrm{NiO}$, probably the most exhaustively studied material among the transition metal oxides. The Raman spectrum of $\mathrm{NiO}$ is well known $[10,11]$. It consists of several two-phonon features and one two-magnon line at nearly $1500 \mathrm{~cm}^{-1}[10,11]$. The optical properties of $\mathrm{NiO}$ have been extensively investigated $[12,13,16]$. Below $3.5 \mathrm{eV}$ the absorption spectrum shows bands which corresponds to intra $\mathrm{d}-\mathrm{d}$ transitions of the $\mathrm{Ni}^{2+}$ ions [12] (thus equivalent to the transitions discussed above for $\alpha-\mathrm{Fe}_{2} \mathrm{O}_{3}$ and $\mathrm{FeBO}_{3}$ ). At higher energies the absorption rises steeply and the first peak in the reflectivity is found at $4 \mathrm{eV}[12,13,16]$. There is no consensus in the literature as to the assignment of this absorption edge. According to Adler and Feinleib [13] it is due to $3 \mathrm{~d}^{8} \rightarrow 3 \mathrm{~d}^{7} 4 \mathrm{~s}$ transitions. This type of excitation is expected to exhibit some electric dipole character, due to covalency of the selectrons with the anion wave function [13]. Other authors $[12,14]$ have assigned this edge to a charge transfer $\mathrm{O}^{2-}$ to $\mathrm{Ni}^{2+}$ transition. Photoconductivity measurements, on the other hand, have been interpreted by assigning the edge to a transition between neighbouring $\mathrm{Ni}^{2+}$ ions: $2 \mathrm{Ni}^{2+} \rightarrow \mathrm{Ni}^{3+}+\mathrm{Ni}^{1+}[15]$.

We have measured the dependence of the Raman scattering cross section for two magnons and for the two-phonon peaks below and nearly above the absorption edge. Our results are shown in fig. 2 . All the second order phonon structures resonate near the edge whereas the two-magnons do not resonate at all. This result indicates that the transition responsible for the absorption edge is not $3 \mathrm{~d}^{8} \rightarrow 3 \mathrm{~d}^{7} 4 \mathrm{~s}$. The transition 


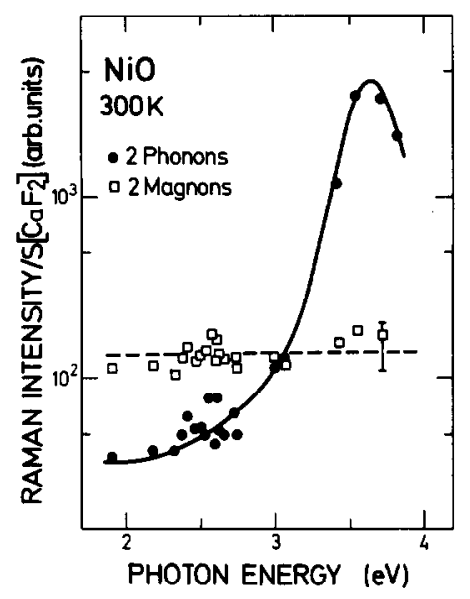

Fig. 2. Dependence of the integrated intensity of the twomagnon Raman peak (squares) and the $2 \mathrm{LO}(\mathrm{L})$ phonon peak (circles) normalized to that of $\mathrm{CaF}_{2}$. The assignment for the two-phonon structure was taken from ref. [10].

$2 \mathrm{Ni}^{2+} \rightarrow \mathrm{Ni}^{3+}+\mathrm{Ni}^{1+}$ is unlikely to explain the observed absorption coefficient $\left(5 \times 10^{5} \mathrm{~cm}^{-1}\right)[12,13,16]$ since it occurs between second nearest neighbours. It could not produce resonant two-magnon scattering either.

From these facts we conjecture that the edge is due to the charge transfer $\mathrm{O}(2 \mathrm{p}) \rightarrow \mathrm{Ni}(3 \mathrm{~d})$ transitions.

\section{Acknowledgements}

We would like to thank Prof. S. Hüfner for illuminating discussions and Dr. G. Güntherodt for the critical reading of the manuscript.

\section{References}

[1] B. Andlauer, O.F. Schirmer and J. Schneider, Solid State Commun. 13 (1973) 1655; B. Andlauer, J. Schneider and W. Wettling, J. Appl. Phys. 10 (1976) 189 and references therein.

[2] B. Andlauer et al., to be published.

[3] N. Koshizuka, T. Okuda and M. Udagawa, J. Phys. Soc. Japan 37 (1974) 354.

[4] T.R. Hart, S.B. Adams and H. Temkin, in Light Scattering in Solids, eds. M. Balkansky, R.C.C. Leite and S.P.S. Porto (Flammarion Sciences, Paris, 1976).

[5] R. Loudon, Advan. Phys. 17 (1968) 243; P.A. Fleury and R. Loudon, Phys. Rev. 166 (1968) 514 and references therein.

[6] T.P. Martin, R. Mcrlin, D.R. Huffman and M. Cardona, Solid State Commun. 22 (1977) 565.

[7] J.J. Krebs, Phys. Rev. 135 (1964) 396.

[8] P.C. Bailey, J. Appl. Phys. 31 (1960) 39S.

[9] J.J. Krebs and W.G. Maisch, Phys. Rev. B4 (1971) 757.

[10] C.H. Perry, E. Anastassakis and J. Sokoloff, Indian J. Pure and App. Phys. 9 (1971) 930.

[11] R.E. Dietz, G.I. Parisot and A.E. Meixner, Phys. Rev. B4 (1971) 2302.

[12] R. Newman and R.M. Chrenko, Phys. Rev. 114 (1959) 1507.

[13] D. Adler and J. Feinleib, Phys. Rev. B2 (1970) 3112.

[14] K.H. Johnson, R.P. Messner and J.W.D. Connolly, Solid State Commun. 12 (1973) 313; K.S. Kim, J. Elec. Spectry. 3 (1974) 217.

[15] Ya. M. Ksendzov and I.A. Drabkin, Sov. Phys. S.S. 7 (1965) 1519.

[16] R.J. Powell and W.E. Spicer, Phys. Rev. B2 (1970) 2182. 